\title{
Closing the gap in a regional health service in NSW: a multistrategic approach to addressing individual and institutional racism
}

\section{Hunter New England Health Aboriginal and Torres Strait Islander Strategic Leadership Committee}

Corresponding authors.Emails: Tony.Martin@hnehealth.nsw.gov. au and Michael.DiRienzo@hnehealth.nsw.gov.au

\begin{abstract}
Building a culturally safe and respectful organisation that genuinely addresses individual and institutional racism is a substantial and complex undertaking. Achieving this outcome requires sustained commitment and a comprehensive strategy, including the active involvement of Aboriginal stakeholders. This paper describes the journey of a large regional health organisation in NSW. A multistrategic approach is broadly described, with three strategies explored in depth. These are: staff education and training; leadership; and consultation, negotiation and partnerships. Challenges are discussed in the context of promising progress and an ongoing commitment to this important organisational goal.
\end{abstract}

\section{Terminology}

In accordance with NSW Health policy, the term 'Aboriginal' is used throughout this document to include Aboriginal and Torres Strait Islander peoples. No disrespect is intended towards our Torres Strait Islander staff, patients or communities, whose contribution to this important project is gratefully acknowledged.

The inequality between the health and wellbeing outcomes of Aboriginal and non-Aboriginal Australians is well documented, and closing this gap is the focus of many plans and strategies. ${ }^{1-5}$ While the issues contributing to this health disparity are complex, there is no doubt that inequitable access to health services is a concern. ${ }^{5-8}$ The racism that many Aboriginal people experience when they access the health system contributes to the low levels of access to health services. ${ }^{6-11}$ These issues also affect our capacity to recruit and retain Aboriginal staff, and to provide them with a safe and respectful working environment. ${ }^{12-14}$

Racism occurs at both the individual and institutional level (Box 1). ${ }^{15}$ Individually, the attitudes, words and practices of health services staff can have a profound effect on Aboriginal clients. ${ }^{10,11}$ This issue is often the focus of workforce strategies such as cultural awareness training. ${ }^{10,13,16}$ While such strategies are appropriate and important, a broader, critical issue is the degree to which racism becomes embedded and perpetuated at the organisational level. ${ }^{6,7,9}$ Institutional racism results in a systemic failure of the organisation to meet the needs of Aboriginal peoples. Discrimination and inequities in the structure of policies, services and funding are readily identifiable but it is often the more subtle manifestations of racism throughout an organisation that go unrecognised. ${ }^{6,7,9}$

All mainstream health programs and services must be appropriate, acceptable and accessible to all Australians. To achieve effective and sustainable change, a systematic organisational approach is required to address racism at both the individual and institutional levels. This paper describes the response of a large regional health organisation in NSW to institutional racism.

\section{Addressing individual and institutional racism}

Hunter New England Health (HNE Health) has the largest Aboriginal population of any NSW Local Health District. The executive team is committed to closing the gap in health and wellbeing outcomes between Aboriginal and non-Aboriginal peoples as a key strategic objective. ${ }^{17}$ All parts of the service are responsible and accountable for achieving this goal.

Institutional racism, by its very nature, is entrenched throughout an organisation. ${ }^{7}$ To address it effectively requires a complex combination of strategies. Figure 1 presents an overview of the multistrategic approach that has been adopted by HNE Health, with examples of specific initiatives beside each strategy. It should be noted that there are many more initiatives than those shown in Figure 1, and that there is substantial interconnectivity between them. Collectively they contribute to the objectives of culturally competent staff, culturally safe workplaces and culturally respectful health services. 


\section{Box 1. A definition of individual and institutional racism}

Racism in general terms consists of conduct or words or practices which advantage or disadvantage people because of their colour, culture or ethnic origin. In its more subtle form it is as damaging as in its overt form.

Institutional Racism consists of the collective failure of an organisation to provide an appropriate and professional service to people because of their colour, culture or ethnic origin. It can be seen or detected in processes, attitudes and behaviour which amount to discrimination through unwitting prejudice, ignorance, thoughtlessness, and racist stereotyping which disadvantage minority ethnic people.

Source: The McPherson Report: Home Department (UK). The Stephen Lawrence Inquiry: Report of an inquiry by Sir William Macpherson of Cluny. Cm 4262-1. London: The Stationery Office; $1999 .{ }^{15}$

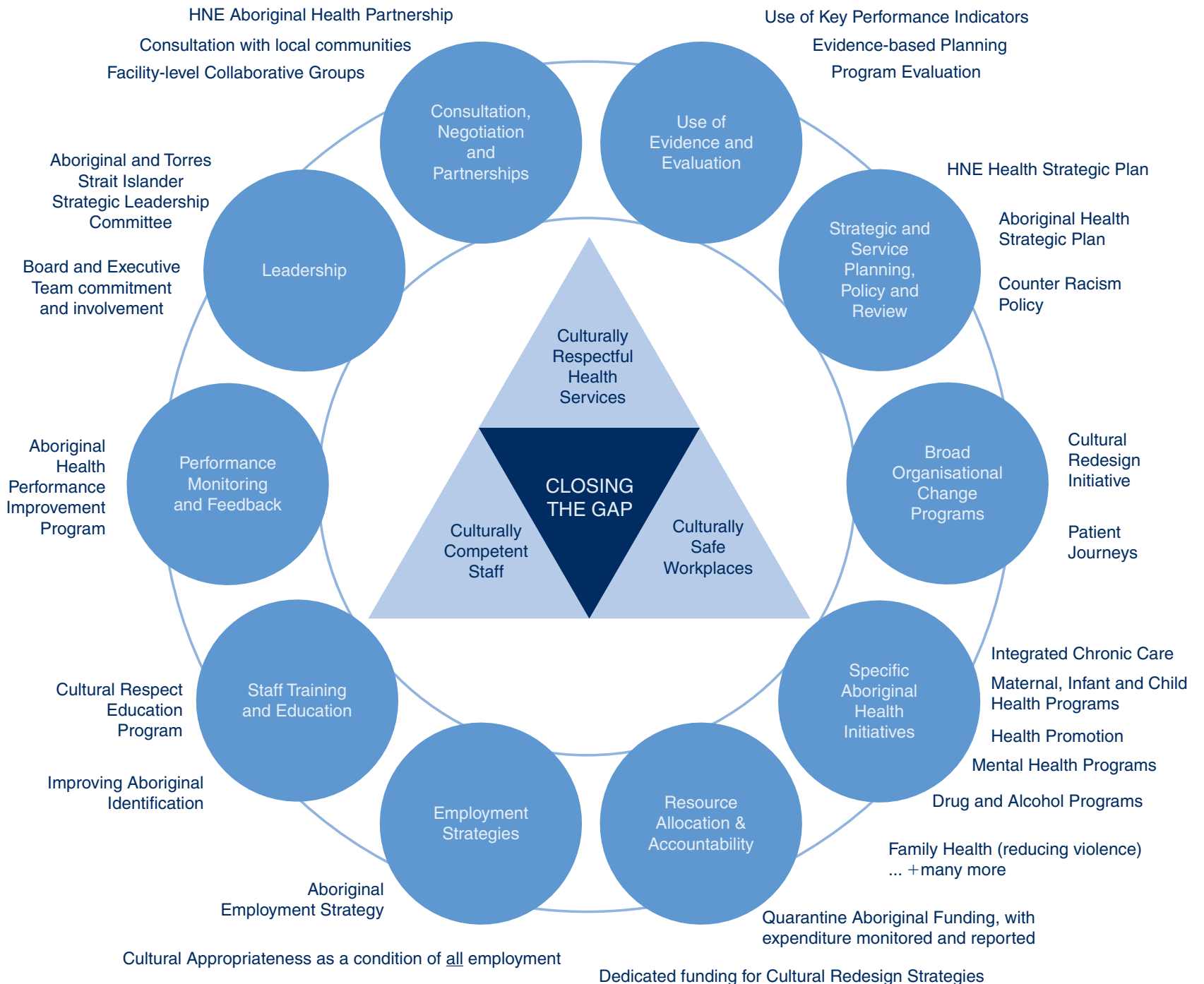

Dedicated funding via EOls to local services

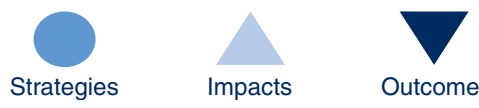

Figure 1. An overview of the multistrategic approach adopted by Hunter New England Health to address individual and institutional racism. 
The strategies described in Figure 1 have evolved over a number of years, and will continue to evolve. In this paper, we focus on three specific strategies: staff education and training; leadership; and consultation, negotiation and partnerships. All three are vital to the overall approach, and were identified as among the earliest priorities for action, from which many of the other strategies would then flow.

\section{Staff training and education: building culturally competent staff}

Health is primarily a people business, and the cultural safety and appropriateness of our services are largely influenced by the cultural competency of our staff. Cultural competence begins with a strong appreciation of the need for cultural respect. ${ }^{18,19}$ To that end, delivery of a Cultural Respect Education Program commenced in 2007.

The approach taken in this Program differs from what is usually taken in cultural awareness training. The Program is deliberately challenging and confronting. Delivered over 2 days, the focus is understanding the meaning of being part of the dominant culture, discussion of white privilege and racism (both individual and institutional) and the impact of dominant culture on the lives of Aboriginal Australians. The Program encourages participants to question their own beliefs, assumptions, life experiences and attitudes..$^{9,20,21}$

The Program was developed and initially delivered by consultants from South Australia - an Aboriginal woman in partnership with a non-Aboriginal person. The consultants delivered the Program to more than 300 staff over 2 years. While this was a great achievement, it was important to develop a more cost-effective strategy to deliver the Program sustainably on a wider scale. Two Cultural Respect Co-facilitators were appointed, and with the support and mentoring of the original consultants, they developed a localised program to be offered within HNE Health. The co-facilitators work in partnership with HNE Health to develop, implement and evaluate the Cultural Respect Education Program, and provide strategic advice about implementing the Program.

Over 1350 HNE Health staff have now attended the workshops. The long-term goal is for all HNE Health staff to attend the training, but with a workforce of over 16000 , the short-term priority is for staff in services with a high contact with Aboriginal people to be trained first.

The reaction from staff has generally been positive, with many describing a profound personal reaction to the difficult content (Box 2). However there have been other reactions; while service managers have reported that the majority of participants return to their workplace with a more culturally respectful approach, some workers have been angry that the organisation forced them to attend, and have struggled to accept or conform to the principles of the
Box 2. Reactions of Hunter New England Health staff to the Cultural Respect Education workshops

$$
\text { Eye-opening ..." }
$$

"This was most profoundly life changing. I have never experienced training which has made me consider my attitudes,

beliefs and behaviours and the impact this has on others ... "

"... Uncomfortable..."

". . . New insight ..."

"I was humbled and felt overwhelmed as I reflected on my previous attitude and service delivery to Aboriginal

communities

training. This response has demonstrated the complexity and depth of the racial issues that must be addressed, and the importance of a multistrategic approach that extends beyond the training itself. Follow-up strategies have included individual counselling, performance improvement and monitoring and, where necessary, more stringent approaches including reference to the HNE Health Code of Conduct, Respectful Workplace Policy and disciplinary procedures. At times, this procedure has required intensive and challenging management processes.

An evaluation of the program commenced in early 2012. It will consider the strengths and gaps in program design and delivery, short and medium-term outcomes, and future directions.

\section{Leadership}

The active support of the highest levels of the organisation is essential. ${ }^{19}$ The Aboriginal and Torres Strait Islander Strategic Leadership Committee is co-chaired by the Director, Aboriginal Health and the Chief Executive of HNE Health. It includes several members of the executive team, HNE Health staff and representatives of partner organisations. Importantly, the Committee has equal numbers of Aboriginal and non-Aboriginal members.

The Aboriginal and Torres Strait Islander Strategic Leadership Committee has provided strong leadership in establishing many of the initiatives shown in Figure 1. For example, the Cultural Redesign Initiative is a service-wide, organisational change program that coordinates a wide range of strategies. The Initiative ensures that the learnings from the Cultural Respect Education Program are translated into meaningful change within the workplace. A range of tools, guidelines and resources are being developed to assist this process, and to build the capacity of the organisation to meet this goal. For example, the Counter Racism Policy Compliance Procedure will assist in the identification and reporting of racism in the workplace, with specific, executive-endorsed processes to investigate and address allegations of workplace racism when they arise. 
Leading by example, the entire HNE Health executive team were among the first to complete the Cultural Respect Education Program. This top-down approach was useful not only to demonstrate commitment, but also to ensure leadership and reinforcement of learnings in the workplace. Managers cannot provide meaningful and informed follow-up, support and management of staff returning from the Program if they have not attended themselves. Therefore it was agreed that senior managers would be prioritised, and that no staff should attend until their immediate line manager had been trained. Over $90 \%$ of senior managers have now completed the training.

Leadership is also vital at the facility level. Facility-led Collaborative Groups involve health service management and Aboriginal and Torres Strait Islander staff to provide ongoing discussion and review of relevant policy, planning, service delivery and resource allocation.

\section{Consultation, negotiation and partnerships}

The concept of partnership is another vital aspect for ensuring mainstream services can meet the needs of Aboriginal and Torres Strait Islander peoples more effectively. Partnership implies genuine and meaningful input and influence at all levels of the organisation, and a shared approach to decision making that is respectful of and responsive to Aboriginal cultures. ${ }^{3,19,22}$ HNE Health has formal partnerships with Aboriginal stakeholders, including the HNE Aboriginal Health Partnership with Aboriginal Community Controlled Health Services. There are numerous other collaborative groups, including the Aboriginal and Torres Strait Islander Strategic Leadership Committee, and specific committees and working groups for many of the initiatives described in Figure 1. The aim of these groups is to provide effective leadership and ongoing advice on health policy, planning, service delivery and resource allocation.

Perhaps most importantly, partnerships rely on trust (Box 3). ${ }^{22}$ To build this trust, HNE Health is committed to improving consultation mechanisms, both internally

Box 3. Reflections on the importance of trust when working in partnership

Partnerships between organisations start from trust between individuals. It is important for organisations to have a clear agreement about their partnership, what they will put into it and what they will work towards together. Partnership agreements are important for continuity and for conflict resolution, but as one participant in this research said: 'It's what is in the guts of the relationship that will produce outcomes, not the agreement papers'.

Source: Davies J. Walking together, working together: Aboriginal research partnerships. Alice Springs: Desert Knowledge Cooperative Research Centre; $2007 .^{22}$ with Aboriginal staff, and externally with Aboriginal communities and organisations. Besides the Aboriginal Health Impact Statement, consultation guidelines are also being developed; they will engage Aboriginal staff and community representatives, including Elders and organisations, to ensure cultural appropriateness. Cultural appropriateness will not be limited to the planning stages of the initiatives, but will be continued on throughout all stages of development, with regular communication of progress and identification of concerns that can then be addressed together.

\section{Learnings so far}

While progress is difficult to measure in quantitative terms, there have been significant learning outcomes. As previously mentioned, some staff have reacted negatively to the challenging nature of the Cultural Respect Education Program. While the intensive ramifications of managing this response at the individual level have already been discussed, broader organisational consequences remain. The resulting conflict may inadvertently have a detrimental effect on the cultural safety of the workplace, both for Aboriginal colleagues and Aboriginal people accessing the health service. Careful and at times stringent management has been required to address this consequence of the Program.

The undertakings described within this paper all stem from the fundamental acknowledgment that individual and institutional racism do exist within our health service. Bringing this issue so openly to the forefront can and does result in a greater emphasis on these difficult issues, and a need for increased and improved response by management. The program has increased the expectations of Aboriginal staff members that all issues of racism will be dealt with appropriately. While this is the intention, the varying experience, understanding and conviction of individual managers in regard to racism result in inconsistent approaches to managing such issues. Many matters escalate rapidly for resolution by the executive team, involving substantial time commitment. It is important to equip managers to be able to effectively address all sensitive, complex and difficult to resolve issues and, above all, to acknowledge that long-term organisational change cannot be achieved without substantial and sustained effort by many people.

The executive team recognises these challenges and is determined to improve its management to give Aboriginal staff, patients and community members confidence that the health service has zero tolerance to racism. Investment in mediation and team building in some teams that have a significant percentage of Aboriginal staff has created greater understanding of what working in partnership involves, and how best to manage tensions safely when they arise. 
Despite the challenges, feedback from managers throughout the service indicates a growing commitment to the principles of cultural redesign, and a sense of pride that the organisation has commenced this journey.

\section{Conclusions}

Building a culturally safe and respectful organisation that genuinely addresses racism and establishes effective partnerships with Aboriginal peoples is a long and complex undertaking. The many strategies and initiatives described here demonstrate the scope of work that is necessary to achieve sustainable organisational change. After several years of intensive and proactive work, the foundations have been laid by providing strong, consistent and visible leadership, building the cultural competency of staff, and committing to genuine shared decision making. The challenge is to maintain the focus and to build on the work that has started as we continue to redesign our health services to better meet the needs of Aboriginal peoples and truly close the gap in health outcomes between Aboriginal and nonAboriginal Australians.

\section{Acknowledgments}

In respect to the wide range of individuals and communities who have had input into this important work, individual authors have not been listed. The working group for this paper wishes to acknowledge our local Aboriginal communities and staff, the consultants involved in the original Cultural Respect Education Program and the members of their local communities who contributed to it, and members of local leadership groups that have supported this undertaking, notably the Aboriginal and Torres Strait Islander Strategic Leadership Committee, Cultural Respect Education Steering Committee, Aboriginal Employment Strategy Steering Committee and the HNE Health Aboriginal Health Partnership Committee.

\section{References}

1. Commonwealth of Australia. National Strategic Framework for Aboriginal and Torres Strait Islander Health 2003-2013: Australian Government Implementation Plan 2007-2013. Canberra: Commonwealth of Australia; 2007.

2. Council of Australian Governments. National Partnership Agreement on Closing the Gap in Indigenous Health Outcomes: An agreement between the Commonwealth of Australia and the States and Territories. Canberra: Commonwealth of Australia; 2009.

3. National Aboriginal Community Controlled Health Organisation. Close the Gap: Solutions to the Indigenous Health Crisis Facing Australia. Braddon (Canberra): Oxfam Australia; 2007.

4. Health NSW. National Partnership Agreement on Closing the Gap in Indigenous Health Outcomes: Implementation Plan - Jurisdiction: New South Wales. North Sydney: NSW Department of Health; 2008.

5. Pink B, Allbon P. The Health and Welfare of Australia's Aboriginal and Torres Strait Islander Peoples. Canberra: Australian Institute of Health and Welfare; 2008.
6. Australian Medical Association. Institutionalised Inequity: Not Just a Matter of Money. Australian Medical Association Report Card Series 2007.

7. Henry BR, Houston S, Mooney GH. Institutional racism in Australian healthcare: a plea for decency. Med J Aust 2004; 180: $517-20$

8. Larson A, Gillies M, Howard PJ, Coffin J. It's enough to make you sick: the impact of racism on the health of Aboriginal Australians. Aust N Z J Public Health 2007; 31: 322-9. doi:10.1111/j.1753-6405.2007.00079.x

9. Bond CJ. A culture of ill health: public health or Aboriginality? Med J Aust 2005; 183: 39-41.

10. Durey A. Reducing racism in Aboriginal health care in Australia: where does cultural education fit? Aust NZJ Public Health 2010; 34(Suppl 1): S87-92. doi:10.1111/j.1753-6405.2010.00560.x

11. Gallaher G, Ziersch A, Baum F, Bentley M, Palmer C, Edmondson $\mathrm{W}$ et al. In our own backyard: urban health inequities and Aboriginal experiences of neighbourhood life, social capital and racism. Adelaide: Flinders University; 2009.

12. Australian Government Department of Families. Housing, Community Services and Indigenous Affairs. Strategic Plan for Implementation: Aboriginal and Torres Strait Islander Workforce Strategy 2010-2012. Canberra: Australian Government; 2010.

13. NSW Health. Respecting the difference: an Aboriginal cultural training framework for NSW Health. North Sydney: NSW Ministry of Health; 2011.

14. Council of Australian Governments. National Indigenous Reform Agreement: An agreement between the Commonwealth of Australia and the States and Territories. Canberra: Commonwealth of Australia; 2009.

15. Home Department (UK). The Stephen Lawrence Inquiry: Report of an inquiry by Sir William Macpherson of Cluny. Cm 4262-1. London: The Stationery Office; 1999.

16. Betancourt J, Green A, Carrillo J. Cultural competence in health care: emerging frameworks and practical approaches. The Commonwealth Fund; 2002.

17. HNE Health. HNE Health Strategic Plan 2012-2015 (unreleased draft). New Lambton: Hunter New England Local Health District; 2012.

18. Australian Health Ministers' Advisory Council Standing Committee on Aboriginal and Torres Strait Islander Health Working Party. Cultural Respect Framework for Aboriginal and Torres Strait Islander Health. Adelaide: Department of Health South Australia; 2004.

19. National Health and Medical Research Council. Cultural competency in health: a guide for policy, partnerships and participation. Canberra: Commonwealth of Australia; 2006.

20. Gollan S. The reasons for why . . . In: Anderson L, editor. Bedtime Stories for Tired Therapists. Adelaide: Dulwich Centre Publications; 2005.

21. Tamasese K, Waldegrave C. Cultural and gender accountability in the 'Just Therapy' approach. J Fem Fam Ther 1994; 5: 29-45. doi:10.1300/J086v05n02_03

22. Davies J. Walking together, working together: Aboriginal research partnerships. Alice Springs: Desert Knowledge Cooperative Research Centre; 2007. 